\title{
1 T cell receptor (TCR) repertoire analysis reveals a highly conserved 2 TCR repertoire in a bilateral tumor mouse model
}

4 Mikiya Tsunoda $^{\mathrm{a}, \mathrm{c}}$, Hiroyasu Aoki ${ }^{\mathrm{a}, \mathrm{b}, \mathrm{c}}$, Haruka Shimizu ${ }^{\mathrm{a}}$, Shigeyuki Shichino ${ }^{\mathrm{a}}$, Kouji Matsushima ${ }^{\mathrm{a}}$, 5 Satoshi Ueha ${ }^{a,{ }^{*}}$

$6 \quad{ }^{a}$ Division of Molecular Regulation of Inflammatory and Immune Diseases, Research Institute for

7 Biomedical Sciences, Tokyo University of Science

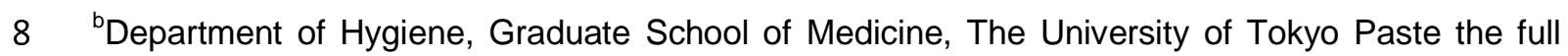
9 affiliation list here.

$10{ }^{\mathrm{c}}$ These authors contributed equally; Mikiya Tsunoda, Hiroyasu Aoki

12 *corresponding; Satoshi Ueha, Ph.D

13 Email: ueha@rs.tus.ac.jp

15 Author Contributions: M.T., H.A., S.S., K.M., and S.U. designed research; M.T., H.A., and H.S. 16 performed research; M.T., H.A., and S.U. analyzed data; M.T., H.A., and S.U. wrote the initial 17 draft of manuscript; All the authors participated in writing the final manuscript.

19 Keywords: Cancer immunotherapy, immunomonitoring, TCR sequencing, overlap analysis.

21 This PDF file includes:

Main Text

23

Figures 1 to 4 


\section{$24 \quad$ Abstract}

25 Temporal analysis of the T cell receptor (TCR) repertoire has been used to monitor treatment-

26 induced changes in antigen-specific $T$ cells in patients with cancer. However, the lack of

27 experimental models that allow temporal analysis of the TCR repertoire in the same individual in

28 a homogeneous population limits the understanding of the causal relationship between changes

29 in TCR repertoire and antitumor responses. A bilateral tumor model, where tumor cells were

30 inoculated bilaterally into the backs of mice, could be used for temporal analysis of the TCR

31 repertoire. This study examined the prerequisite for this strategy: the TCR repertoire is conserved

32 between bilateral tumors with the same growth rate. Bilateral tumors with equivalent size and

33 draining lymph nodes (dLNs) were collected 13 days after tumor inoculation to analyze the TCR

34 repertoire of $\mathrm{CD}^{+}$and $\mathrm{CD}^{+} \mathrm{T}$ cells. The tumor-infiltrating $\mathrm{T}$ cell clones were highly conserved

35 between the bilateral tumors, and the extent of clonal expansion was equivalent. In addition, the

36 similarity between the bilateral tumors was equivalent to heterogeneity on one side of the tumor.

37 The similarity of the TCR repertoire in the bilateral dLNs was markedly lower than that in the

38 tumor, suggesting that tumor-reactive T cell clones induced independently in each dLN integrated

39 during recirculation and then infiltrated the tumor. These findings suggest that our bilateral tumor

40 model is suitable for temporal monitoring of the TCR repertoire to evaluate temporal and

41 treatment-induced changes in tumor-reactive T cell clones. 


\section{Significance Statement}

43 The bilateral subcutaneous tumor model, where tumor cells were inoculated bilaterally into the

44 backs of mice, is a promising model for temporal analysis of the antitumor response in cancer

45 immunotherapy. This study demonstrated a highly conserved TCR repertoire in bilateral tumors

46 and provided the basis for using a bilateral tumor model for evaluating temporal and treatment-

47 induced changes in tumor-responsive $T$ cell clones in individual mice. In humans, accurate

48 statistical analysis is hampered by various background factors, including cancer type and stage

49 and history of treatment. Moreover, temporal tumor biopsy in patients is highly invasive. Our

50 bilateral tumor model overcomes these clinical issues and is expected to be a valuable tool for

51 the development of novel immune monitoring and therapeutic strategies. 


\section{Introduction}

53 Immune checkpoint inhibitors (ICls) have a significant therapeutic effect in some cancers and

54 have become an important pillar of cancer treatment in recent years $(1,2)$. However, the 55 response rate to $\mathrm{ICl}$ monotherapy is less than $30 \%$ for most types of cancer, and ICls 56 occasionally cause severe immune-related adverse effects in some patients $(3,4)$. Thus, the 57 development of reliable biomarkers that represent tumor-specific immune responses and stratify 58 the responder and non-responder at an early stage is essential to optimize the usage of ICls (5, $596)$.

60 Because ICls suppress tumor growth by enhancing the proliferation and activation of tumor61 specific T cells (7), the efficacy of ICls is closely associated with the strength of tumor-specific T 62 cell responses. These tumor-specific T cells are composed of various tumor-reactive T cell clones 63 with different specificities to tumor-associated antigens $(8,9)$. Antigen specificity of $T$ cell clones 64 is determined by their T cell receptors (TCRs), which are generated by $V(D) J$ recombination in 65 the thymus and are incredibly diverse $(10,11)$. Therefore, global analysis of the collection of 66 TCRs using next-generation sequencing, that is TCR repertoire analysis, can now be applied to 67 monitor tumor-specific $T$ cell responses in patients receiving ICls.

68 Several studies have reported the features of the TCR repertoire in mice treated using 69 immunotherapy. Philip and Rudqvist reported enhanced clonal expansion of tumor-infiltrating T 70 cells in mice treated with an anti-cytotoxic $\mathrm{T}$ lymphocyte-associated protein 4 monoclonal 71 antibody $(12,13)$. We reported that $\mathrm{CD}^{+} \mathrm{T}$ cell clones with a TCR identical to tumor-infiltrating $\mathrm{T}$ 72 cells could be detected in the peripheral blood and draining lymph node (dLN) of tumor-bearing 73 mice (14). Based on the cancer-immunity cycle, where tumor-specific $T$ cells are primed in the $74 \mathrm{dLN}$ and infiltrate into the tumor via lymph-blood circulation (15), these "overlapping" $\mathrm{T}$ cell 75 clones seem to reflect the mobilization of $\mathrm{T}$ cells into antitumor responses. Consistent with this 76 hypothesis, the diversity and total frequency of $\mathrm{CD}^{+} \mathrm{T}$ cell clones that overlapped between the 77 blood and tumor increased after depletion of $\mathrm{CD} 4^{+}$cells, the degree of which was correlated with 78 antitumor effects in mice and humans $(14,16)$. To validate whether these features of the TCR 
79 repertoire are predictive of the antitumor effect of ICls, an appropriate analysis of the temporal or

80 treatment-induced changes in TCR repertoire is needed. However, temporal analysis of the TCR

81 repertoire of tumor-reactive $\mathrm{T}$ cells has not been reported in murine tumor model, owing to the

82 difficulty in sampling from an individual mouse.

83 Recently, Zemek and Chen reported that the immune microenvironment is similar in bilateral

84 tumor models with comparable tumor growth and that it can be applied to temporal analysis of

85 antitumor immune responses $(17,18)$. To apply this method for temporal analysis of the TCR

86 repertoire, there are important prerequisites: (1) the TCR repertoires of the left and right tumors

87 show similar characteristics; and (2) the same tumor-reactive $\mathrm{T}$ cell clones infiltrate into the

88 bilateral tumors. In this study, we investigated whether the bilateral tumor model is suitable for

89 examining the temporal responses of tumor-reactive T cells in individual mice. 


\section{Results}

\section{$91 \quad$ T cell repertoire of bilateral tumor exhibits similar characteristics.}

92 We used the bilateral tumor model to establish an experimental system for evaluating temporal 93 and treatment-induced changes in tumor-reactive T cell clones. To achieve this goal, the growth 94 rate and clonal T cell responses in bilateral tumors must be similar in individual mice. Therefore, 95 we first examined whether the Lewis lung carcinoma (LLC) tumors inoculated bilaterally into the 96 back of individual mice grew symmetrically. Intratumoral injection of Evans Blue verified that the 97 brachial lymph node became a dLN in the subcutaneous tumors (Fig. 1A). The growth curves of 98 bilateral tumors were symmetrical in individual mice, suggesting that an equivalent antitumor 99 response occurred on both sides of the tumor (Fig. 1B).

100 Next, we investigated the equivalency of T cell clonal responses inside the bilateral tumors. CD4 101 and $\mathrm{CD}^{+} \mathrm{T}$ cells from the tumor and $\mathrm{CD} 4^{+} \mathrm{CD} 44^{\mathrm{hi}}$ and $\mathrm{CD} 8^{+} \mathrm{CD} 44^{\mathrm{hi}} \mathrm{T}$ cells from dLN were sorted 10213 days after tumor inoculation, and their TCR repertoires were analyzed (Fig. 1C and SI 103 Appendix Fig. S1A). Variable-Joining (V/J) segment usage of TCR $\beta$, which is commonly used to 104 characterize individual repertoire, was highly similar between bilateral tumors in the same mouse, 105 but variable among mice (Fig. 1D and E). The proportion of the most abundant T cell clones was 106 also similar between bilateral tumors, suggesting that they contained an equivalent number of 107 expanded clones (Fig. 1F). Consistently, the clonality of the $\mathrm{CD}^{+} \mathrm{T}$ cell repertoire, which 108 represents the extent of clonal expansion, was equivalent between the bilateral tumors (Fig. 1G). 109 In addition, the difference in clonality between the bilateral tumors of the same mouse was 110 significantly smaller than that between different mice (Fig. $1 \mathrm{H})$. A similar trend was observed for 111 the $\mathrm{CD}^{+} \mathrm{T}$ cell repertoire (SI Appendix Fig. S1). These results demonstrated that the global 112 features of the TCR repertoire were similar between the bilateral tumors. 
116 Highly similar features of the TCR repertoire between bilateral tumors suggested that $\mathrm{T}$ cell 117 clones present on one side also exist on the other side. To examine this, we identified $\mathrm{T}$ cell 118 clones that overlapped between the bilateral tumors or different individuals (Fig. 2A). The clones 119 shared between bilateral tumors accounted for approximately $80 \%$ of the $\mathrm{CD}^{+} \mathrm{T}$ cell repertoire. 120 However, T cell clones overlapping between different mice covered only approximately $3 \%$. This 121 result indicated that most of the tumor-infiltrating $\mathrm{CD}^{+} \mathrm{T}$ cell clones were commonly present on 122 both sides of the tumor.

123 Next, we examined whether these overlapping clones expanded equally in bilateral tumors. To 124 this end, we depicted the frequency of each overlapping clone within the right ( $x$-axis) and left ( $y$ 125 axis) tumors as a scatter plot (Fig. 2B). The frequency of clones that had expanded to more than $1261 \%$ was almost equal between the left and right sides. There were some clones whose frequency 127 differed between the left and right sides, but the frequency of these clones was relatively low. 128 Consistent with this finding, the Morisita-Horn similarity index, which reflects the similarity of two T 129 cell repertoires considering the frequency of each shared clone (19), was over 0.9 between the 130 bilateral tumors, whereas it ranged from 0.05 to 0.3 among the tumors in different individuals (Fig. $1312 \mathrm{C}$ ). The $\mathrm{CD}^{+} \mathrm{T}$ cell repertoire also showed similar tendencies (SI Appendix Fig. S2). These 132 results suggest that the majority of $\mathrm{CD}^{+} \mathrm{T}$ cell clones in tumors respond to tumor antigens 133 shared between the bilateral tumors. Altogether, these data showed that the majority of the T cell 134 repertoire of bilateral tumors was composed of shared clones, and these clones expanded to a 135 similar extent

136 in the left and right tumors. Thus, the clonal T cell responses on one side may reflect those on the 137 other side in our bilateral tumor model.

139 Similarity of TCR repertoires between bilateral tumors was equivalent to the similarity 140 within the tumor.

141 Previous studies have shown that there is intratumoral heterogeneity of the TCR repertoire (20). 
142 When extrapolating our bilateral tumor model to a clinical situation, where antitumor immune 143 responses are longitudinally monitored by tumor biopsy, it is important to determine whether the 144 difference in TCR repertoire between the bilateral tumors could be considered equivalent to that 145 within one side of the tumor. To examine this, we divided each tumor into two pieces at the time 146 of collection and analyzed the TCR repertoire independently to estimate intra-tumoral TCR 147 heterogeneity. We then compared the similarity of the TCR repertoire within one side of the tumor 148 and between bilateral tumors (Fig. 3A). The total frequency of $\mathrm{CD}^{+}$overlapping clones between 149 the left and right tumors was approximately $80 \%$, which was equivalent to the frequency of 150 overlapping clones within one side (Fig. 3B). Additionally, scatter plots depicting the frequency of 151 each overlapping clone showed that the variance in frequency of overlapping clones between 152 bilateral tumors was equivalent to that within the tumor (Fig. 3C). Consistently, there was no 153 significant difference in the similarity of the TCR repertoire between the tumor and within the 154 tumor (Fig. 3D). A similar tendency was observed for the CD4 ${ }^{+} \mathrm{T}$ cell repertoire (SI Appendix Fig. 155 S3). These data demonstrated that the differences in TCR repertoires between the bilateral 156 tumors were equivalent to the TCR heterogeneity within each tumor. This also suggested that our 157 bilateral tumor experiments could be considered as an experimental model for temporal 158 monitoring of TCR repertoire using sequential tumor biopsy.

Proportional infiltration of T cell clones into bilateral tumors contributed to the similarity of TCR repertoires.

163 blood circulation via the thoracic duct, and eventually form a blood repertoire. Thus, it is 164 hypothesized that the tumor-reactive clones induced on one side of the dLNs infiltrate into the 165 bilateral tumors at equal probability through the circulation and then proliferate in situ at the same 166 rate (Fig. 4A). To explore this idea, we investigated whether the frequency of overlapping clones 167 between the tumor and its contralateral dLN was equivalent to that of overlapping clones between 168 the ipsilateral ones (Fig. 4A). The total frequency of overlapping clones between contralateral 
$169 \mathrm{dLNs}$ in the tumor was almost the same as that between the ipsilateral ones, and this result 170 supported our idea (Fig. 4B).

171 However, it was unclear whether similar clones were induced in bilateral dLNs. To address this 172 query, we examined the frequency of overlapping clones in the bilateral dLNs and found that it 173 was lower than $10 \%$, which was substantially lower than the overlap between bilateral tumors, 174 although it was higher than the overlap between individuals (Fig. 4C). Because the dLN CD8 ${ }^{+}$ $175 \mathrm{CD} 44^{\mathrm{hi}} \mathrm{T}$ cells may contain a substantial proportion of clones not associated with the tumor, we 176 next examined the TCR repertoire of bilateral dLNs that overlapped with either of the bilateral 177 tumors to enrich tumor-associated clones. In dLN-tumor overlapping clones in the bilateral dLNs, 178 the frequency of left-right overlapping clones between bilateral dLNs increased to approximately $17940 \%$ (Fig. 4D), and the proportion of left-right overlapping clones was approximately 20-40\% 180 (Fig. 4E). In other words, more than 50\% of dLN-tumor overlapping clones were induced only in 181 one of the dLNs. Finally, we investigated whether these dLN-tumor overlapping clones were 182 equally expanded in the bilateral dLNs. Using scatter plots, we showed that the correlation in 183 frequency in dLN-tumor overlapping clones was moderate between the bilateral dLNs $(0.4<\mathrm{r}<$ 184 0.48; Fig. 4F), compared to the correlation between the bilateral tumors $(0.71<r<0.85$; Fig. 2B). 185 The frequency of $\mathrm{CD}^{+} \mathrm{T}$ cell clones overlapping between bilateral dLNs was higher than that of $186 \mathrm{CD}^{+} \mathrm{T}$ cell clones, and the $\mathrm{CD}^{+} \mathrm{T}$ cell repertoire also showed similar tendencies (SI Appendix 187 Fig. S4).

188 Collectively, these results suggested that the tumor-reactive $T$ cell clones induced in the bilateral $189 \mathrm{dLNs}$ were only moderately conserved, and the degree of expansion of the shared clones differed 190 between the bilateral dLNs. Therefore, the proportional infiltration of T cell clones into bilateral 191 tumors through blood circulation contributed to the conserved TCR repertoire. 
193 The bilateral subcutaneous tumor model, where tumor cells were inoculated bilaterally into the 194 backs of mice, is a promising model for temporal analysis of the antitumor response in cancer 195 immunotherapy. In this study we examined the prerequisite for this strategy: the TCR repertoire is 196 conserved between bilateral tumors with the same growth rate. We found that bilateral tumors 197 with similar growth rates contained a highly conserved $\mathrm{CD}^{+}$and $\mathrm{CD} 4^{+} \mathrm{T}$ cell repertoire in our 198 experimental model. Interestingly, the TCR repertoires in bilateral dLNs were less conserved than 199 those in bilateral tumors. These results suggest that T cell clones induced in the bilateral dLNs 200 eventually integrated into a blood repertoire, proportionally infiltrated the bilateral tumors, and 201 proliferated in situ at a similar rate. These findings provide the basis for analyzing temporal and 202 treatment-induced changes in tumor-reactive T cell clones using a bilateral tumor model in mice.

203 Previous reports have examined the relationship between the immune microenvironment before $204 \mathrm{ICl}$ treatment and the antitumor response in bilateral tumor models; Zemeck et al. reported that in 205 bilateral tumor models, the transcriptional signature of tumors before $\mathrm{ICI}$ treatment was different 206 between responders and non-responders (17). Chen and colleagues analyzed tumor-infiltrating $207 \mathrm{CD}^{+} \mathrm{T}$ cells in bilateral tumor models treated with $\mathrm{ICl}$ and reported that non-responders had an 208 exhaustion signature and responders had an activation signature (18). All of these reports 209 suggest that similar immune and antitumor responses are induced in bilateral tumors. Our finding 210 of a highly conserved TCR repertoire in bilateral tumors may explain why similar immune 211 responses and gene expression are induced bilaterally.

212 Although we did not test other mouse tumor models, our bilateral tumor model would be 213 applicable in other tumor models, because the conserved TCR repertoire between the bilateral 214 tumors seems to be dependent on anatomical factors that are absolutely conserved among 215 individuals. Notably, the difference in tumor size may alter the tumor microenvironment, such as 216 the concentrations of chemo-attractants and vascularity, between bilateral tumors, and it may 217 decrease the similarity in TCR repertoire between the bilateral tumors. Verifying the bilateral 218 symmetry of tumor growth or therapeutic response is necessary to evaluate the temporal 
219 changes in the tumor-reactive T cell repertoire using this model.

220 In humans, patient background data, such as cancer type and stage and history of treatment are

221 associated with prognosis (21). Thus, it was difficult to obtain a large cohort of homogeneous

222 patients for statistical analysis. Moreover, temporal tumor biopsy in patients is highly invasive.

223 Considering these difficulties, clinical studies to validate the relationship between TCR repertoire

224 and antitumor effects are limited. We believe that our bilateral tumor mouse model will overcome

225 these barriers and provide valuable suggestions for clinical research.

226 A possible application of this bilateral tumor model is to investigate the features of the TCR 227 repertoire that can predict the therapeutic response to ICls. Considering that the antitumor 228 responses following $\mathrm{ICl}$ therapy vary even among mice with the same genetic background, the 229 specific features of the TCR repertoire may reflect the variance of antitumor immune responses 230 among syngeneic mice. We plan to examine the hypothesis that a large amount of dLN-tumor 231 repertoire overlap before treatment would predict a better therapeutic response to ICls using the 232 bilateral tumor model. Clinically, we observed that an increased frequency of tumor-blood 233 overlapping clones in blood CD8+ $\mathrm{T}$ cells before treatment was associated with a favorable 234 clinical response to PD-1 blockade in gastrointestinal cancer (22). In addition, the bilateral tumor 235 model enables temporal tracking of endogenous $\mathrm{T}$ cell clones in the same mouse, while tumor 236 progression or therapeutic effects continue. We expect that temporal analysis of $\mathrm{T}$ cell clones 237 within bilateral tumors will reveal the kinetics of expansion and contraction of each $\mathrm{T}$ cell clone 238 and the process of exhaustion in the tumor.

239 Overall, this study reported the TCR repertoire analysis of bilateral tumor models, which enables 240 the evaluation of temporal and treatment-induced changes in the tumor-reactive T cell clones. We 241 believe that this novel experimental system will deepen our understanding of the clonal 242 responses of tumor-reactive T cells. 


\section{Materials and Methods}

\section{Mice and cell line.}

245 Eight-week-old female C57BL/6 mice were purchased from Sankyo Labo service corporation inc.

246 (Tokyo, Japan). Lewis lung carcinoma (LLC) was originally provide from the Nihonkayaku (Tokyo, 247 Japan).

\section{Tumor inoculation.}

250 LLC cells $\left(5 \times 10^{5}\right.$ cells /mouse) were inoculated subcutaneously (s.c.) into the bilateral backs of 251 C57BL/6 mice. Tumor diameter was measured twice a week and used to calculate tumor volume $252\left(\mathrm{~mm}^{2}\right)$ according to the following formula [(major axis; $\left.\mathrm{mm}\right) \times$ (minor axis; $\mathrm{mm}$ )]. In some mice, $1 \%$ 253 Evans Blue dye was injected into the tumor $30 \mathrm{~min}$ prior to sacrifice to determine the dLN in our 254 model. All animal experiments were conducted in accordance with institutional guidelines with the 255 approval of the Animal Care and Use Committee of the Tokyo University of Science.

\section{Flow cytometry and Cell Sorting.}

258 Intravascular leukocytes were stained by intravenous injection of FITC-conjugated mAb (3 $259 \mu \mathrm{g} /$ mouse) against CD45.2 (clone 104) three minutes before sacrifice (23). Tumor was equally 260 divided into two parts and processed individually. Each tumor was cut into small fragments and 261 digested for 45 minutes at $37^{\circ} \mathrm{C}$ with $0.1 \%$ collagenase (FUJIFILM Wako, Osaka, Japan). The 262 cells were then subjected to density separation with 40\% Percoll PLUS (Cytiva, Marlborough, 263 MA) and leukocytes were recovered from the bottom layer. ACK buffer was used to lyse red 264 blood cells. The extracted dLN was cut into small fragments and mashed on a cell strainer. The 265 cell number was determined using Flow-Count fluorospheres (Beckman Coulter, San Diego, CA) 266 and a CytoFLEX flow cytometer (Beckman Coulter). Cells were then stained with a mix of Fc 267 Block (anti-mouse CD16/CD32 mAb; clone 2.4G2, BioXcell) and fluorophore-conjugated anti- 
268 mouse mAbs as indicated in Table S1. After enrichment of $\mathrm{T}$ cells with magnetic separation, $269 \mathrm{CD}^{+}$and $\mathrm{CD} 4^{+} \mathrm{T}$ cells from the tumor and $\mathrm{CD} 8^{+} \mathrm{CD} 44^{\mathrm{hi}}$ and $\mathrm{CD} 4^{+} \mathrm{CD} 44^{\mathrm{hi}} \mathrm{T}$ cells from the dLN 270 were sorted using FACS Aria II or Aria III (BD Biosciences, San Jose, CA). Nonviable cells were 271 excluded from the sorting gate based on forward and side scatter profiles and propidium iodide 272 staining. intravascular staining-CD45.2 positive cells were also excluded. Purity of sorted cells 273 was always over 95\%. Data were analyzed using FlowJo software (version 10.5.3; BD 274 Biosciences). TCR library construction and sequencing.

277 TCR libraries were prepared on purified T cells lysed in lysis buffer [1\% LiDS, $100 \mathrm{mM}$ Tris- HCl 278 (pH 7.5), $500 \mathrm{mM} \mathrm{LiCl}$, and $10 \mathrm{mM}$ EDTA]. PolyA RNAs were isolated according to a previous 279 report with some modifications (GSE110711).To perform reverse transcription and template280 swiching, beads were then suspended in $10 \mu \mathrm{L}$ of RT mix [1× First Strand buffer (Thermo Fisher 281 Scientific), $1 \mathrm{mM}$ dNTP, $2.5 \mathrm{mM}$ DTT (Thermo Fisher Scientific), $1 \mathrm{M}$ betaine (Sigma-Aldrich), 9 $282 \mathrm{mM} \mathrm{MgCl}, 1 \mathrm{U} / \mu \mathrm{L}$ RNaseln Plus Rnase Inhibitor (Promega, Madison, WI), $10 \mathrm{U} / \mu \mathrm{L}$ Superscript II 283 (Thermo Fisher Scientific), and $1 \mu \mathrm{M}$ of i5-TSO], and incubated for $60 \mathrm{~min}$ at $42^{\circ} \mathrm{C}$ and 284 immediately cooled on ice. Beads were washed once with $\mathrm{B} \& \mathrm{~W}-\mathrm{T}$ buffer [5 mM Tris- $\mathrm{HCl}(\mathrm{pH} 7.5)$, $2851 \mathrm{M} \mathrm{NaCl}, 0.5 \mathrm{mM}$ EDTA, and 0.1\% Tween-20], and once with Tris- $\mathrm{HCl}$ ( $\mathrm{pH}$ 8.0). To amplify the 286 TCR cDNA containing complementarity determining region 3 (CDR3), nested PCR of the TCR 287 locus was performed as follows. Beads containing cDNA was resuspended with the $25 \mu \mathrm{L}$ of first 288 PCR mixture [0.4 $\mu \mathrm{M}$ of primers (i5, Trac_ex, and Trbc_ex), and 1x KAPA Hifi Hotstart ReadyMix 289 (KAPA Biosystems, Wilmington, MA, \#KK2602)], and the thermal cycling was performed as 290 following condition: denaturation at $95^{\circ} \mathrm{C}$ for $3 \mathrm{~min}, 5$ cycles of denaturation for $20 \mathrm{sec}$ at $98^{\circ} \mathrm{C}$, 291 annealing for $15 \mathrm{sec}$ at $65^{\circ} \mathrm{C}$ and extension for $30 \mathrm{sec}$ at $72^{\circ} \mathrm{C}$, followed by a final extension at $29272^{\circ} \mathrm{C}$ for $2 \mathrm{~min}$. Then, $2.5 \mu \mathrm{L}$ of first PCR product was mixed with the $22.5 \mu \mathrm{L}$ of second PCR 293 mixture [0.35 $\mu \mathrm{M}$ of primers (i5_2nd and i7-BC_mTrbc), and 1x KAPA Hifi Hotstart ReadyMix], 294 and the thermal cycling was performed under the same condition as first PCR. The second-PCR 
295 products were purified by an Agencort AM Pure XP kit (Bexkman-Coulter, CA, \#A63881) at a

$296 \quad 0.7: 1$ ratio of beads to sample, and eluted with $20 \mu \mathrm{L}$ of Tris- $\mathrm{HCl}(\mathrm{pH} 8.0)$. To amplify TCR library

297 and add adaptor sequence for next generation sequencer, the third PCR was performed as

298 follows. $5 \mu \mathrm{L}$ of purified second PCR product was mixed with the $20 \mu \mathrm{L}$ of third PCR mixture [0.4

$299 \mu \mathrm{M}$ of primers (i5-BC and i7-BC), and 1x KAPA Hifi Hotstart ReadyMix (KAPA Biosystems,

300 Wilmington, MA, USA, \#KK2602)], and the thermal cycling was performed under the same

301 condition as first PCR, excepted for the number of cycles (23 cycles). The third-PCR products

302 were purified as second PCR. The products were pooled and then purified and subjected to dual

303 size selection using ProNex size-selective purification system (Promega) and eluted with $25 \mu \mathrm{L}$ of

304 Tris- $\mathrm{HCl}(\mathrm{pH}$ 8.5). Final TCR libraries, whose lengths were about 600 base pairs were sequenced

305 using an Illumina Novaseq 6000 S4 flowcell (67 bp read 1 and 140 bp read 2) (Illumina, USA).

306 Only read2 contained the sequence regarding the definition of $\mathrm{T}$ cell clones.

308 Data processing of TCR sequencing.

309 Adapter trimming and quality filtering of sequencing data were performed using Cutadapt-3.2 (24)

310 and PRINSEQ-0.20.4 (25). Sequencing data were processed by MiXCR-3.0.5 (26). In MiXCR,

311 Filtered reads were aligned to reference mouse TCR V/D/J sequences registered in the

312 international ImMunoGeneTics information system with the following parameters: -starting-

313 material=rna, -5-end=no-v-primers-, -3-end=c-primers, $\quad$-adapters=no-adapters,

314 vParameters.geneFeatureToAlign=VTranscript, -vjAlignmentOrder=JThenV. Then, identical

315 sequences were assembled and grouped in clones with PCR and sequencing error correlation

316 with the following parameters: -badQualityThreshold=15, -separateByV=true, - separateByJ=true,

317 -only-productive=true, -region-of-interest=CDR3. The Variable (V) and Joining (J) segment of

318 TCRs were represented in IMGT gene nomenclature.

319 List of final clones were analyzed by VDJtools-1.2.1 (27). Sequencing reads of sample was

320 normalized to the six times of cell count in each sample by "DownSample" command of VDJtools. 
321 T-cell clones were determined as TCR reads with the same TCR V segment, $\mathrm{J}$ segment and

322 CDR3 nucleotide sequence. TCR repertoires of divided tumors were pooled other than Figure 3.

323 The processed data have been deposited in NCBI GEO under the accession GSE174225.

325 Evaluating the feature of TCR repertoire and the extent of overlap between repertoires.

$326 \mathrm{~V} / \mathrm{J}$ segment usage plots of the TCR repertoire of bilateral tumors were generated by 327 "PlotFancyVJUsage" command of VDJtools. Principle component analysis of V and $\mathrm{J}$ segment 328 usage were performed based on the frequency of each $\mathrm{V}$ or $\mathrm{J}$ segment using prcomp fuction of $\mathrm{R}$ 329 (version 3.6.0). The 1 - Pielou index was used to evaluate the clonality of TCR repertoire, which 330 was calculated using the formula: $1-\sum_{i=1}^{n} p_{i} \log _{e}\left(p_{i}\right) / \log _{e}(n)$ where $p_{i}$ is the frequency of clone 331 i for a sample with $\mathrm{n}$ unique clones. The Morisita-Horn index was used to estimate the similarity of 332 TCR repertoire between bilateral tumors, which was calculated using the formula:

$$
C_{H}=\frac{2 \sum_{i=1}^{S} x_{i} y_{i}}{\left(\frac{\sum_{i=1}^{S} x_{i}^{2}}{X^{2}}+\frac{\sum_{i=1}^{S} y_{i}^{2}}{Y^{2}}\right) X Y}
$$

333 where $x_{i}$ is the number of clones $i$ in the total $\mathrm{X}$ reads of one sample, $y_{i}$ is the number of clones $i$

334 in the total $\mathrm{Y}$ reads of another sample, and $\mathrm{S}$ is the number of clones.

335 The frequency of OL clones between samples is calculated by the geometric mean of the 336 frequencies within each sample.

\section{Statistical analysis.}

339 Statistical analyses were performed using GraphPad Prism (ver8) software (GraphPad Software, 340 La Jolla, CA). Two-sided paired Student's t-test was run on the comparison of frequency of dLN341 Tumor OL clones between ipsilateral and contralateral ones. Ordinary one-way analysis of 342 variance was run to compare the clonality of TCR repertoires between bilateral tumors and 343 between individuals. All other experimental data were analyzed using two-sided unpaired 
344 Student's t-test. Asterisks to indicate significance corresponding to the following: n.s., not 345 significant $(\mathrm{P}>0.05),{ }^{*} \mathrm{P} \leq 0.05,{ }^{* *} \mathrm{P} \leq 0.01,{ }^{* * *} \mathrm{P} \leq 0.001,{ }^{* * *} \mathrm{P} \leq 0.0001$.

\section{Acknowledgments}

348 This work was supported by the Japan Society for the Promotion of Science under Grant Number

34920281832 and 17929397. We would like to thank Y. Hara for advice in cell sorting, staff of RIBS

350 animal facility for supporting maintenance of animals, member of IGT. Inc., and J. Yasuda for 351 expert technical assistance in TCR repertoire analysis, Editage (www.editage.com) for English 352 language editing. 


\section{References}

1. L. Galluzzi, T. A. Chan, G. Kroemer, J. D. Wolchok, A. López-Soto, The hallmarks of successful anticancer immunotherapy. Sci. Transl. Med. 10, 1-15 (2018).

2. A. Ribas, J. D. Wolchok, Cancer immunotherapy using checkpoint blockade. Science (80-. ). 359 (2018).

3. J. Y. Kim, et al., Hyperprogressive disease during anti-PD-1 (PDCD1) / PD-L1 (CD274) therapy: A systematic review and meta-analysis. Cancers (Basel). 11 (2019).

4. M. A. Postow, R. Sidlow, M. D. Hellmann, Immune-Related Adverse Events Associated with Immune Checkpoint Blockade. N. Engl. J. Med. 378 (2018).

5. S. L. Topalian, J. M. Taube, R. A. Anders, D. M. Pardoll, Mechanism-driven biomarkers to guide immune checkpoint blockade in cancer therapy. Nat. Rev. Cancer 16 (2016).

6. G. V. Masucci, et al., Validation of biomarkers to predict response to immunotherapy in cancer: Volume I - pre-analytical and analytical validation. J. Immunother. Cancer 4 (2016).

7. H. O. Alsaab, et al., PD-1 and PD-L1 checkpoint signaling inhibition for cancer immunotherapy: mechanism, combinations, and clinical outcome. Front. Pharmacol. 8 (2017).

8. A. Pasetto, et al., Tumor- and neoantigen-reactive T-cell receptors can be identified based on their frequency in fresh tumor. Cancer Immunol. Res. 4, 734-743 (2016).

9. A. Gros, et al., PD-1 identifies the patient-specific CD8+ tumor-reactive repertoire infiltrating human tumors. J. Clin. Invest. 124 (2014).

10. E. Rosati, et al., Overview of methodologies for T-cell receptor repertoire analysis. BMC Biotechnol. 17 (2017).

11. X. Liu, J. Wu, History, applications, and challenges of immune repertoire research. Cell Biol. Toxicol. 34 (2018).

12. H. Philip, et al., A T cell repertoire timestamp is at the core of responsiveness to CTLA-4 blockade. iScience 24, 102100 (2021).

13. N. P. Rudqvist, et al., Radiotherapy and CTLA-4 blockade shape the tcr repertoire of tumor-infiltrating t cells. Cancer Immunol. Res. 6, 139-150 (2018).

14. H. Aoki, et al., TCR repertoire analysis reveals mobilization of novel CD8+T cell clones into the cancer-immunity cycle following anti-CD4 antibody administration. Front. Immunol. 10 (2019).

15. D. S. Chen, I. Mellman, Oncology meets immunology: The cancer-immunity cycle. Immunity 39, 1-10 (2013).

16. K. Shitara, et al., First-in-human phase 1 study of IT1208, a defucosylated humanized anti-CD4 depleting antibody, in patients with advanced solid tumors. J. Immunother. cancer 7, 195 (2019).

17. R. M. Zemek, et al., Bilateral murine tumor models for characterizing the response to immune checkpoint blockade. Nat. Protoc. 15 (2020).

18. I. X. Chen, et al., A bilateral tumor model identifies transcriptional programs associated with patient response to immune checkpoint blockade. Proc. Natl. Acad. Sci. U. S. A. 117 (2020).

19. J. Chiffelle, et al., T-cell repertoire analysis and metrics of diversity and clonality. Curr. Opin. Biotechnol. 65, 284-295 (2020). 
397 20. D. V. Yuzhakova, et al., Measuring Intratumoral Heterogeneity of Immune Repertoires. Front. Oncol. 10, 1-13 (2020).

399 21. J. J. Havel, D. Chowell, T. A. Chan, The evolving landscape of biomarkers for checkpoint inhibitor immunotherapy. Nat. Rev. Cancer 19, 133-150 (2019).

401 22. H. Aoki, et al., Transient Depletion of CD4 + Cells Induces Remodeling of the TCR Repertoire in Gastrointestinal Cancer . Cancer Immunol. Res. (2021) https:/doi.org/10.1158/2326-6066.cir-20-0989. in press.

404 23. K. G. Anderson, et al., Intravascular staining for discrimination of vascular and tissue

24. M. Martin, Cutadapt removes adapter sequences from high-throughput sequencing reads.

25. R. Schmieder, R. Edwards, Quality control and preprocessing of metagenomic datasets.

26. D. A. Bolotin, et al., MiXCR: Software for comprehensive adaptive immunity profiling. Nat.

27. M. Shugay, et al., VDJtools: Unifying Post-analysis of T Cell Receptor Repertoires. PLoS Comput. Biol. 11, 1-16 (2015). 


\section{Figure}

$415 \quad$ Figure. 1.

A

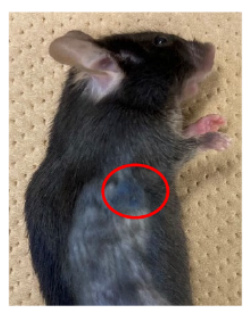

C

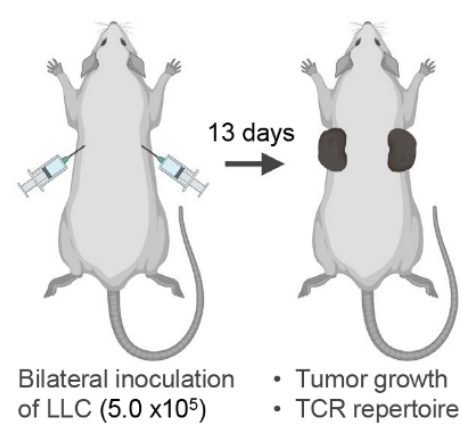

B
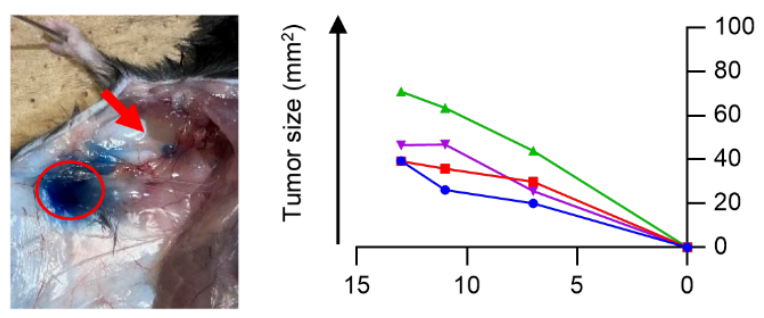

Days after tumor inoculation

D

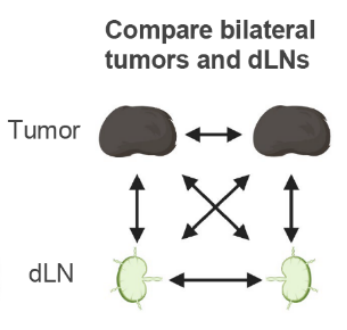

mouse2

mouse1
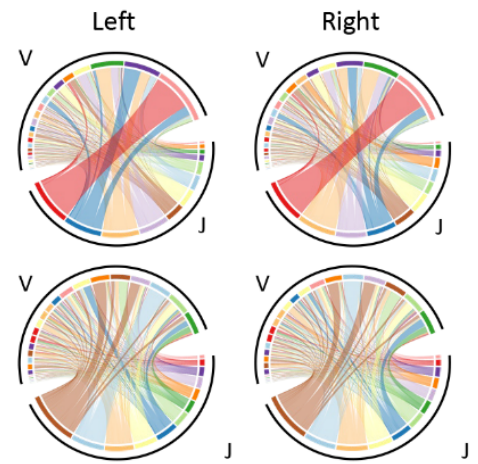

$E$

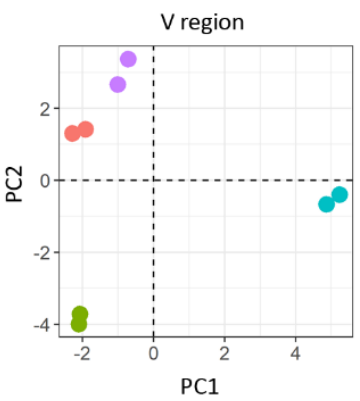

G

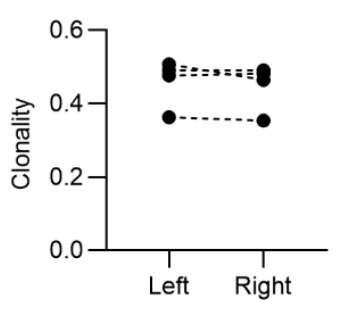

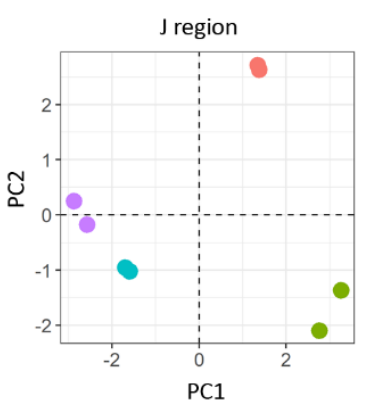

$\mathrm{H}$

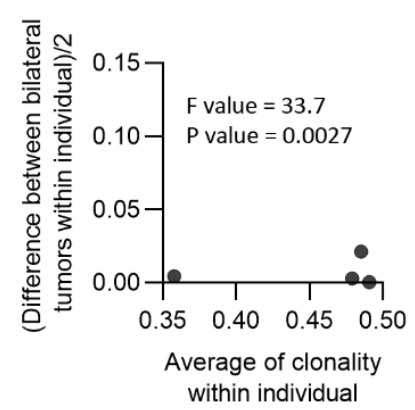

$\mathrm{F}$

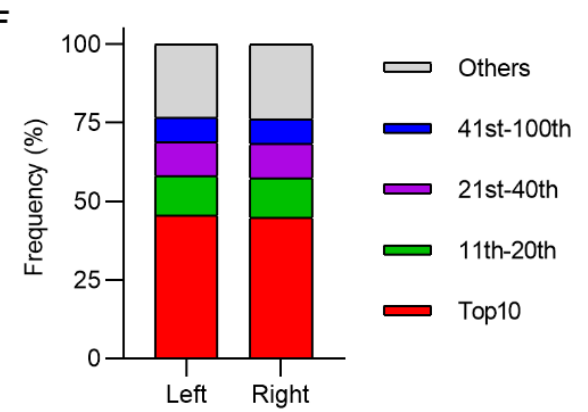

417 Figure. 1. Characteristics of $\mathrm{CD}^{+} \mathrm{T}$ cell repertoire in the bilateral tumor. (A) Visualization of 418 draining lymph node (dLN) in our bilateral tumor model. 1\% Evans Blue dye was injected into the 
419 tumor (red circle) 30 min prior to euthanasia. Brachial LN (arrow) was stained by Evans Blue, 420 indicating this $\mathrm{LN}$ became a dLN. (B) Growth curve of the tumor inoculated bilaterally on the 421 backs. Lines of the same color indicate left and right tumors of the same mouse $(n=4)$. (C) 422 Experimental design. (D) V/J segment usage plots of the bilateral $\mathrm{CD}^{+} \mathrm{T}$ cell repertoires. 423 Ribbons connecting the $\mathrm{V}$ and $\mathrm{J}$ segments are scaled by the corresponding $\mathrm{V} / \mathrm{J}$ pair frequency. 424 (E) Principal component analysis of $\mathrm{V}$ and $\mathrm{J}$ segment usage of tumor-infiltrating $\mathrm{CD} 8^{+} \mathrm{T}$ cells. 425 Points of the same color indicate left and right tumors of the same individual. (F) Frequency of 426 abundant $\mathrm{CD}^{+} \mathrm{T}$ cell clones in the tumor. $\mathrm{CD} 8^{+} \mathrm{T}$ cell clones in the left and right tumor were 427 categorized into five classes based on their rank in each repertoire: top $10,11^{\text {th }}-20^{\text {th }}, 21^{\text {st }}-40^{\text {th }}$, $42841^{\text {st }}-100^{\text {th }}$, and others. The total frequency of clones in each class is shown $(n=4)$. (G) Clonality 429 of the $\mathrm{CD}^{+} \mathrm{T}$ cell repertoire of the left and right tumor. $(\mathrm{H})$ Homoscedasticity plot for variance of 430 clonality in individual mice. The X-axis represents the average clonality of bilateral tumors in each 431 individual. The $\mathrm{Y}$-axis represents variance of clonality between different bilateral tumors within 432 individuals. Sum of squares within mouse $=9.7 \times 10^{-4}$; sum of squares between mice $=2.4 \times 10^{-2}$; 433 degree of freedom within mouse $=4$; degree of freedom between mice $=3$. 


\section{$434 \quad$ Figure. 2.}

A

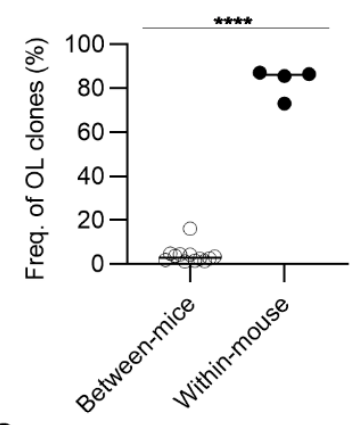

C

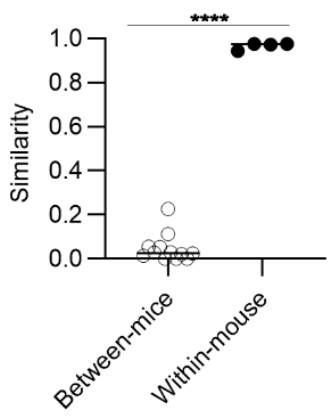

B
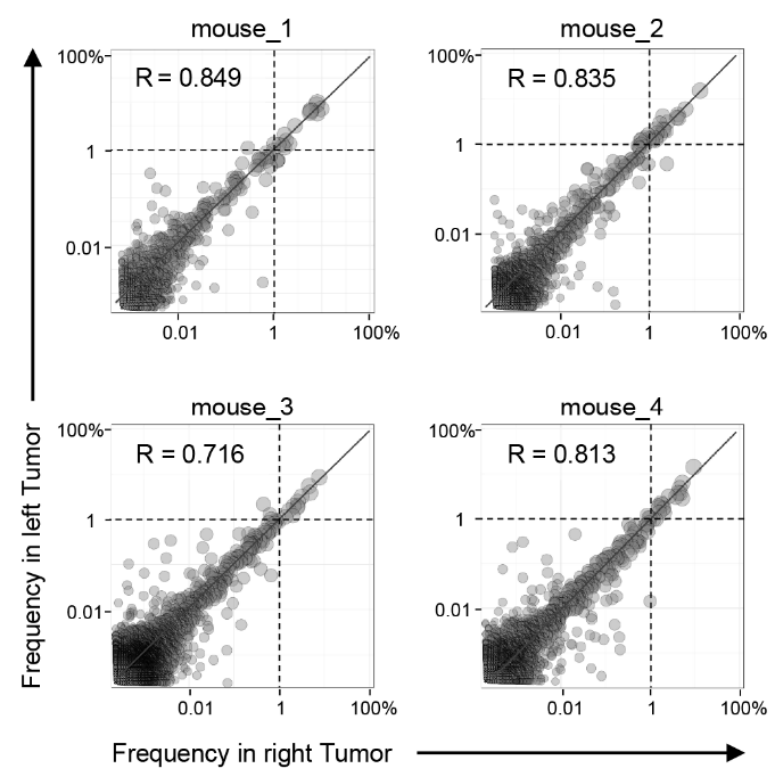

Frequency in right Tumor

Figure. 2. Clonal composition of $\mathrm{CD}^{+} \mathrm{T}$ cell repertoires in bilateral tumors. (A) Comparison of the

437 frequency of overlapping clones in tumors between and within mice (between mice, $\mathrm{n}=4 \times 3$;

438 within mice, $\mathrm{n}=4$ ). (B) Scatter plot of $\mathrm{CD}^{+} \mathrm{T}$ cell clones from bilateral tumors. Each plot 439 represents a single clone with indicated frequency in the left (X-axis) and right tumors (Y-axis).

440 The dotted line indicates a frequency of $1 \%$. (C) Comparison of the similarity of the tumor 441 repertoires between and within mice. Mean; Two-sided unpaired Student's t-test $(\mathrm{A}$ and $\mathrm{C}) ;{ }^{* * *} \mathrm{P}$ $442 \leq 0.0001$. 
$443 \quad$ Figure. 3.

A

Each tumor was divided into two pieces.

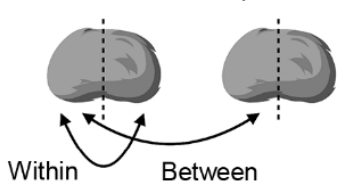

Compare similarity of TCR repertoire.

C

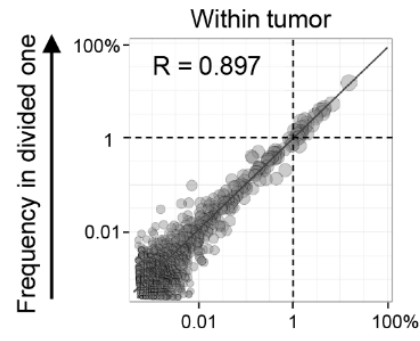

B
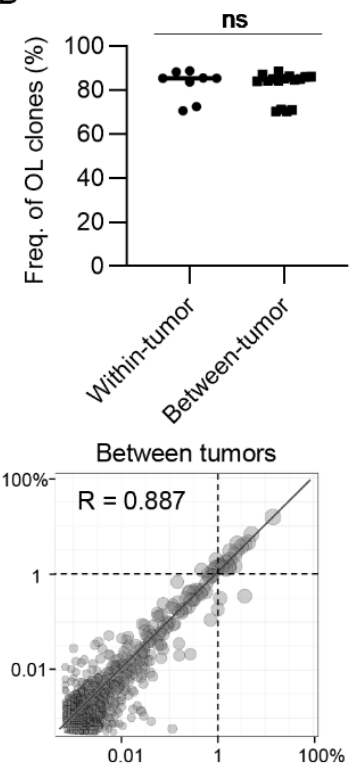

D

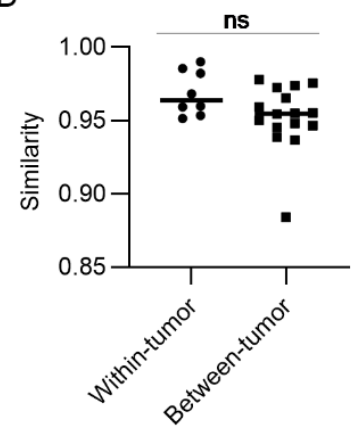

Frequency in divided one

445 Figure. 3. The extent of difference of $\mathrm{CD}^{+} \mathrm{T}$ cell repertoires between bilateral tumors. (A)

446 Schematic diagram of the analysis. (B) Comparison of the frequency of the clones overlapped

447 between divided same side tumors and bilateral tumor fragments $(n=4)$. (C) Scatter plot of CD8 ${ }^{+}$

448 T cell clones from bilateral tumors. Each plot represents a single clone with indicated frequency in

449 each tumor fragment. The dotted line indicates a frequency of $1 \%$. (D) Comparison of the

450 similarity of the tumor repertoires between divided same tumors and bilateral tumor fragments. (B

451 and D) Within-tumor, $n=4 \times 2$; between-tumor, $n=4 \times 4$; Mean; Two-sided unpaired Student's t-

452 test; ns, non-significant. 
Figure. 4.

A

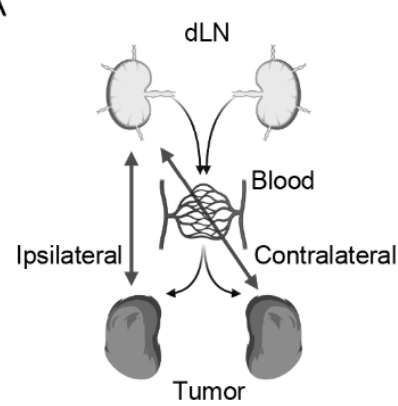

D

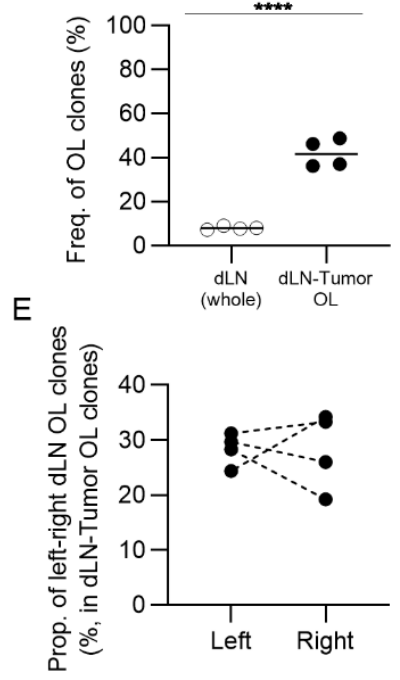

B

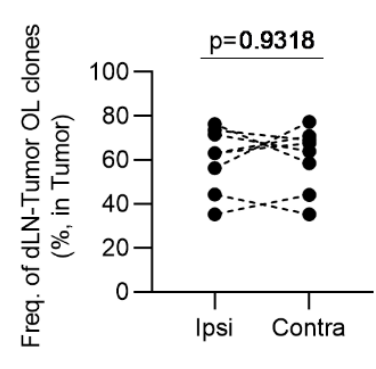

$\mathrm{F}$

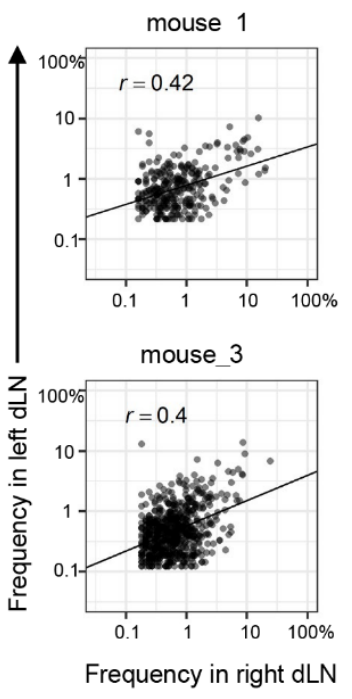

C
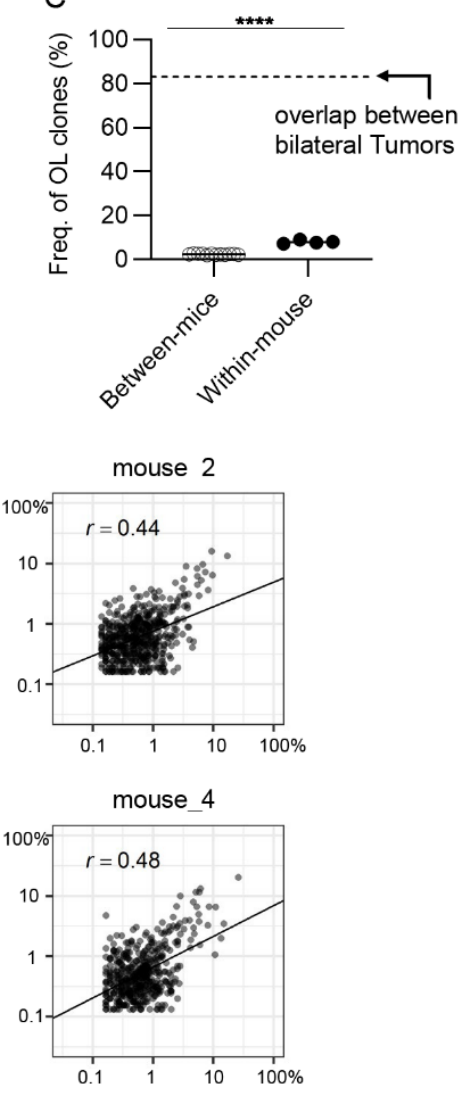

455 Figure. 4. The similarity of $\mathrm{CD}^{+} \mathrm{T}$ cell repertoires in the bilateral dLNs. (A) Schematic diagram of 456 the hypothesis that $\mathrm{T}$ cell clones induced in $\mathrm{dLN}$ infiltrate into tumors evenly through blood 457 circulation. (B) The frequency of the clones overlapped between the dLN and its ipsilateral and 458 contralateral tumor. Ipsi, ipsilateral; Contra, contralateral. $n=4 \times 2$. (C) Comparison of the 459 frequency of overlapping clones in dLN between and within mice (between mice, $n=4 \times 3$; within 460 mice, $n=4)$. Dotted line indicates the frequency of clones overlapped between bilateral tumors 461 within the mouse. (D) Comparison of the frequency of left-right overlapping clones within whole $462 \mathrm{dLNs}$ and dLN-tumor overlapping repertoire $(n=4)$. (E) The proportion of left-right overlapping 463 clones within the dLN-tumor OL repertoires. (F) Scatter plot of left-right overlapping clones within 464 the dLN-tumor OL repertoires. Each plot represents a single clone with indicated frequency in 
bioRxiv preprint doi: https://doi.org/10.1101/2021.05.13.443732; this version posted May 14, 2021. The copyright holder for this preprint (which was not certified by peer review) is the author/funder. All rights reserved. No reuse allowed without permission. each dLNs. Mean; Two-sided unpaired Student's t-test; ns, non-significant. ${ }^{* \star *} \mathrm{P} \leq 0.0001$. 